\title{
TOXIC EPIDERMAL NECROLYSIS IN A SYSTEMIC LUPUS ERYTHEMATOSUS PATIENT
}

Germana Ribeiro Araujo Carneiro de Lucena , „, Daniel Viana da Silva e Silva', Igor Beltrão Duarte Fernandes', Milvia Maria Simões e Silva Enokihara', Edgard Torres dos Reis Neto'

1.Universidade Federal de São Paulo, São Paulo (SP), Brazil.

*Corresponding author: germanalucena.ufpb@gmail.com

\section{BACKGROUND}

Toxic epidermal necrolysis (TEN) is a rare and potentially fatal situation provoked by drugs, usually between 1 to 8 weeks after the begin of medication. The majority of cases happens after using a high-risk medication, such as allopurinol, anticonvulsants or non-steroidal anti-inflammatory. Hydroxychloroquine is rarely associated with TEN, some cases have already been described even with fatal outcome.

\section{CASE REPORT}

A 22-year-old female with a recent diagnosis of systemic lupus erythematosus was on prednisone $60 \mathrm{mg}$, hydroxychloroquine 400 $\mathrm{mg} /$ day and mycophenolate sodium $360 \mathrm{mg} 12 / 12$ hours. She presented to the São Paulo Hospital with multiple hyperemic lesions on trunks, superior and inferior members, with epidermic detachment on face, multiple painful oral and vaginal ulcers, vasculitic lesions on hands and foots, just as purulent vaginal discharge. All symptoms started one day before hospital admission. At presentation she was on septic shock, with necessity of mechanical ventilation, antibiotics and vasoactive drugs. Mucocutaneous lesions were assessed as TEN and a skin biopsy was performed. Hydroxychloroquine was the most probable drug implied and was promptly discontinued. Since, she had vasculitic palmoplantar lesions, lupic cutaneous activity causing TEN could not be excluded and treatment aiming to resolve was immediately initiated. Urinalysis revealed dysmorphic erythrocytes and subnephrotic proteinuria, normal creatinine was observed on blood test. She also had leukocytosis: 18.610, C reactive protein: $66 \mathrm{mg} / \mathrm{dL}$ and a high procalcitonin: 5,5 ng/mL. Skin biopsy showed interface dermatitis, multiple eosinophilic apoptotic bodies and necrosis with detachment of the epidermis, confirming TEN. The patient received methylprednisolone $1 \mathrm{mg} / \mathrm{kg}$ IV and $2 \mathrm{~g} / \mathrm{kg}$ of intravenous immunoglobulin for TEN, acral vasculitis and lupic nephritis, concomitant to antibiotics. No further immunosuppression could be made, since she was infected. After initial treatment, there were great improvement on mucocutaneous lesion and initial good response on sepsis. Unfortunately, she evolved with ventilator-associated pneumonia and ended up dying of infectious complication three weeks after her admission.

\section{CONCLUSION}

Despite being a rare complication, TEN and other hypersensitivity reactions have been associated with hydroxychloroquine, with potentially fatal outcomes. The severity of those conditions reinforces the importance to maintain surveillance in all patient who initiates this medication. 